\title{
11. Friedens- und Sicherheitspolitik
}

\author{
Xavier Tschumi Canosa
}

\section{OpenEdition \\ Journals}

Electronic version

URL: http://journals.openedition.org/sjep/161

DOI: 10.4000/sjep.161

ISSN: 1663-9677

\section{Publisher}

Institut de hautes études internationales et du développement

\section{Printed version}

Date of publication: 1 avril 2006

Number of pages: 181-196

ISBN: 2-88247-062-2

ISSN: $1660-5926$

\section{Electronic reference}

Xavier Tschumi Canosa, «11. Friedens- und Sicherheitspolitik», Schweizerisches Jahrbuch für Entwicklungspolitik [Online], 25-1 | 2006, Online erschienen am: 17 Mai 2010, abgerufen am 07 September 2020. URL : http://journals.openedition.org/sjep/161 ; DOI : https://doi.org/10.4000/sjep. 161

(c) The Graduate Institute 


\section{Friedens- und Sicherheitspolitik ${ }^{*}$}

D IE SCHWEIZ hat die Friedenspolitik zu einem grundlegenden Element ihrer Aussenpolitik gemacht. Die schweizerische Friedenspolitik setzt einen deutlichen Schwerpunkt auf die zivile Konfliktbearbeitung und die Förderung der Menschenrechte. Diese Aktivitäten werden in einem vom Bundesrat angenommenen Jahresbericht vorgestellt. Die schweizerische Diplomatie - insbesondere die auf das Konzept der Menschlichen Sicherheit ausgerichtete Politische Abteilung IV des EDA - ist dabei der Hauptakteur. Unter den aktuellen Schwerpunktthemen der Friedenspolitik der Schweiz sind der Kampf gegen die Verbreitung illegaler Kleinwaffen und der Kampf gegen Personenminen zu nennen.

Die Sicherheitspolitik der Schweiz definiert sich über die Beteiligung des Landes an der Organisation für Sicherheit und Zusammenarbeit in Europa (OSZE), an der Partnerschaft für den Frieden (PfP) und am Euro-Atlantischen Partnerschaftsrat (EAPC), wobei die beiden letztgenannten Institutionen an die NATO gebunden sind. Die Beteiligung der Schweiz an den beiden Partnerschaften ist Gegenstand jährlicher Berichte; der zuletzt erschienene Bericht deckt das Jahr 2004 ab. Ebenfalls auf Jahresbasis, jedoch mit prospektiver Ausrichtung, präsentiert die Schweiz ihr individuelles Partnerschaftsprogramm, das sie im folgenden Jahr auf militärischer und ziviler Ebene in der Schweiz wie auch in der euro-atlantischen Region zu verfolgen beabsichtigt.

\subsection{Friedenspolitik}

\subsubsection{Bericht der Schweiz über die zivile Konfliktbearbeitung und Menschenrechtsförderung (2004)}

Der Bundesrat hat am 18. Mai 2005 den Bericht 2004 über Massnahmen zur zivilen Konfliktbearbeitung und Menschenrechtsförderung angenommen ${ }^{1}$. Grundlage für diese Tätigkeiten, für die vor allem das Eidgenössische Departement für auswärtige Angelegenheiten (EDA) zuständig ist, ist das neue Bundesgesetz über Massnahmen zur zivilen Friedensförderung und Stärkung der Menschenrechte, das am 1. Mai 2004 in Kraft getreten ist. Die Kosten, die durch den ersten entsprechenden Mehrjahreskredit gedeckt wurden, betrugen 2004 45,85 Millionen Franken.

Dahrbuch 2005, Nr. 1, Kapitel 11, Neues Gesetz über die zivile Friedensförderung, S. 182.

Der Bericht ist in vier Teile gegliedert: Der erste Teil betont die Verankerung der Massnahmen zur zivilen Konfliktbearbeitung und Menschenrechtsförderung

\footnotetext{
Von Xavier Tschumi Canosa, wissenschaftlicher Mitarbeiter am IUED.

Eidgenössisches Departement für auswärtige Angelegenheiten (EDA), Bericht 2004 über Massnahmen zur zivilen Konfliktbearbeitung und Menschenrechtsförderung, vom Bundesrat am 18. Mai 2005 angenommen, 22 S., einsehbar auf der Website des EDA unter: <www.eda.admin.ch> >Themen $>$ Menschliche Sicherheit >Publikationen.
} 
im Aussenpolitischen Bericht 2000 des Bundes sowie ihren Beitrag zur Verwirklichung der Millenniums-Entwicklungsziele (MDG). Ausserdem wird daran erinnert, dass diese Massnahmen der Schweiz direkte Vorteile bringen für die nationale Sicherheit, den internationalen Einfluss und die Wahrung der schweizerischen Interessen, die Migrationsprävention, aber auch für die Aussenwirtschaft und die Investitionsförderung. Der erste Teil des Berichts hält fest, dass diese Massnahmen bewusst auf bestimmte Regionen und Themen konzentriert werden, je nachdem, in welchem Ausmass „ein Engagement den aussenpoliti-

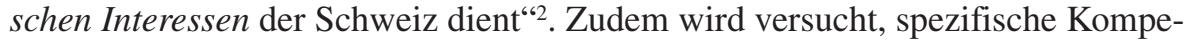
tenzen zu mobilisieren und die Aktionen anderer Akteure der internationalen Gemeinschaft auf sinnvolle Weise zu ergänzen.

Der zweite Teil des Berichts behandelt die schweizerischen Aktivitäten der zivilen Konfliktbearbeitung im Jahr 2004, auf die insgesamt 87 Prozent der 45,85 Millionen Franken, d.h. knapp 40 Millionen Franken entfielen. Diplomatische Demarchen, gute Dienste oder Konfliktbearbeitungsprojekte wurden kombiniert; hinzu kommt die Mobilisierung des Schweizerischen Expertenpools für die zivile Friedensförderung (SEF). Der SEF entsandte im Jahr 2004200 Mitglieder (ein Viertel davon Frauen) zu Einsätzen in 25 Ländern, wo sie sich in den Bereichen Menschenrechte, Rechtsstaat, Wahlbeobachtung oder Unterstützung der Zivilpolizei engagierten.

Unter den im Bericht hervorgehobenen Themenschwerpunkten sind der Kampf gegen die illegale Verbreitung von Kleinwaffen und der Kampf gegen Personenminen zu nennen (siehe unten, 11.3. und 11.4.). Daneben hat sich die Schweiz für andere prioritäre Themen engagiert, die zusammen mit den oben genannten den Bereich der Menschlichen Sicherheit definieren, dem die diplomatischen und operationellen Aktionen der Schweiz für zivile Friedensförderung und Menschenrechte zuzuordnen sind (siehe unten).

Der dritte Berichtsteil ist den Aktivitäten der Menschenrechtsförderung gewidmet. Die entsprechenden Ausgaben beliefen sich im Jahr 2004 auf knapp 6 Millionen Franken (siehe unten).

[D] Jahrbuch 2006, Nr. 1, Kapitel 9, 9.5. Menschenrechtspolitik.

Der vierte Teil des Bericht betrifft die Organisation der Umsetzung der Friedensförderung und der Menschenrechte, mit einer verstärkten Verantwortung des EDA (besonders der Politischen Abteilung IV für Menschliche Sicherheit), die eng mit anderen Bundesstellen zusammenarbeitet ${ }^{3}$.

\subsubsection{Schweizerische Tätigkeiten im Bereich der zivilen Friedensförderung und der Stärkung der Menschenrechte}

In diesem traditionellen Tätigkeitsbereich war die Schweiz 2005 mit guten Diensten und Vermittlung in Friedensprozessen diskret aber aktiv an verschiedenen Schauplätzen tätig, insbesondere im Sudan, in Kolumbien, im Nahen Osten, in Sri Lanka, Somalia, Burundi, in Mazedonien und im Kosovo.

\footnotetext{
Ibid., S. 3.

Insbesondere mit der Direktion für Entwicklung und Zusammenarbeit (DEZA), der Direktion für Völkerrecht (DV) und dem Zentrum für internationale Sicherheitspolitik (ZISP) des EDA sowie mit bestimmten Stellen des Eidgenössischen Departements für Verteidigung, Bevölkerungsschutz und Sport (VBS) und des Eidgenössischen Justiz- und Polizeidepartements (EJPD).
} 
Mit Konfliktbearbeitungsprogrammen wurde ausserdem ein Beitrag zur Behandlung von Fragen betreffend Machtaufteilung, Dezentralisierung und Demokratisierung, Vergangenheitsbewältigung, Medien und bewaffnete Konflikte, Achtung der Menschenrechte und Schutz der Zivilbevölkerung in bewaffneten Konflikten geleistet. Die Schweiz war 2005 insbesondere im Nahen Osten präsent - mit ihrer Unterstützung der Genfer Initiative, der zivilen Beobachtungsmission „Temporary International Presence in the City of Hebron“ und mit Projekten zur Förderung der menschlichen Sicherheit -, im Balkan, in Sri Lanka - mit einem komplexen Programm zur Erleichterung der Verhandlungen für den Frieden und zur Unterstützung der Zivilgesellschaft -, in Nepal, im südlichen und zentralen Afrika (Angola, Simbabwe, Demokratische Republik Kongo), im Sudan, im Südkaukasus, in Kolumbien und in Guatemala.

Daneben setzte sich die Schweiz für die Förderung der Menschenrechte ein, insbesondere mit den Dialogprozessen, die sie mit China (seit 1991), mit dem Iran (seit 2003) und mit Vietnam (seit 2005) pflegt. Im Rahmen der Vereinten Nationen sind zudem drei schweizerische Initiativen im Jahr 2005 hervorzuheben: der Vorschlag der Schaffung eines Menschenrechtsrates in den laufenden Beratungen über die institutionelle Reform der UNO (der Vorschlag wurde vom Generalsekretär der Vereinten Nationen aufgegriffen); die Einbringung einer Resolution mit dem Titel „Droits de l'homme et justice de transition“ im Rahmen der Menschenrechtskommission, die mit der Unterstützung von rund fünfzig Staaten verabschiedet wurde; und schliesslich die Initiative zur Menschenrechtslage in Nepal, welche zur Annahme einer verurteilenden Resolution führte, es aber vor allem erlaubte, die Grundlagen eines Abkommens über die Eröffnung eines Büros des UN-Hochkommissariats für Menschenrechte zu legen, das die Menschenrechtssituation beobachten und die Regierung über Massnahmen zur Verbesserung der Lage beraten soll.

\subsubsection{Friedenseinsätze von Schweizer Militärs im Ausland}

Im Bericht des Bundesrates über die Sicherheitspolitik der Schweiz 2000 (SIPOL B 2000) $)^{4}$ werden die drei Hauptaufträge der Schweizer Armee definiert: Beiträge zur Friedensförderung und zur Krisenbewältigung im internationalen Rahmen, die Raumsicherung und Verteidigung sowie subsidiäre Einsätze zur Prävention und Bewältigung existenzieller Gefahren. Das Armeeleitbild $\mathrm{XXI}^{5}$, das diese Aufträge beinhaltet, diente als Grundlage für die Reform der Schweizer Armee, die seit Februar 2004 abgeschlossen ist. Seitdem ist ein Prozess zur Optimierung der Reform im Gange, der die Gefahrenentwicklung berïcksichtigen und den finanziellen Rahmenbedingungen Rechnung tragen soll.

Die Beteiligung von Schweizer Armeeangehörigen an internationalen friedensfördernden Einsätzen wird vom Kompetenzzentrum SWISSINT des VBS geleitet. 2005 waren rund 300 freiwillige Militärangehörige im Ausland tätig, die Hälfte davon innerhalb der Swiss Company im Kosovo (SWISSCOY).

4 Bundesrat, Sicherheit durch Kooperation. Bericht des Bundesrates an die Bundesversammlung über die Sicherheitspolitik der Schweiz (SIPOL B 2000), vom 7. Juni 1999 (BB1 1999 7657).

5 Bundesrat, Bericht des Bundesrates an die Bundesversammlung über die Armee und die Militärverwaltung (,Armee XXI“), vom 24. Oktober 2001 (BB1 2002 901). 
Gemäss dem Beschluss des Bundesrates vom 11. Mai 2005 sollte das VBS ab 2008 in der Lage sein, gleichzeitig 500 Armeeangehörige für solche Aufträge einzusetzen.

Seit 1999 lösen sich die Kontingente der SWISSCOY im Kosovo in aufeinanderfolgenden Friedenseinsätzen von jeweils sechs Monaten ab. Der Einsatz der SWISSCOY in der Kosovo-Friedenssicherungstruppe (KFOR, unter NATOKommando) wurde vom Bundesparlament im Juni 2005 um weitere drei Jahre bis 2008 verlängert. Das 11. Kontingent beendete seinen Auftrag im April 2005, das 12. im Oktober 2005. Diese Kontingente bestehen aus höchstens 220 Mitgliedern mit einem geringen Frauenanteil (19 im 13. Kontingent). Sie sind dem österreichischen Kontingent vor Ort zwecks Zusammenarbeit zugewiesen, ihm aber nicht unterstellt. Seit Oktober 2002 sind sie zu Selbstverteidigungszwecken bewaffnet.

Daneben beteiligt sich die Schweiz seit Dezember 2004 mit 20 Schweizer Armeeangehörigen an der Krisenbearbeitungsoperation der Europäischen Union in Bosnien-Herzegowina (EUFOR - Operation Althea). Das am 22. Dezember 2004 unterzeichnete Abkommen zwischen der Schweiz und der Europäischen Union regelt die Einzelheiten der Beteiligung.

Dahrbuch 2005, Nr. 1, Kapitel 11, 11.1.1. Einsatz des Schweizer Militärs in Bosnien-Herzegowina, S. 181-182.

Der EUFOR (European Union Force) vor Ort mangelt es an Lufttransportkapazitäten. Seit Juni 2004 bereits wurden von der Europäischen Union mehrere Gesuche um die Bereitstellung solcher Kapazitäten an die Schweiz gerichtet. Der Bund konnte diese Gesuche im Dezember 2004, als der Einsatz von Schweizer Militärs in der EUROFOR vom Bundesparlament bewilligt wurde, noch nicht positiv beantworten. Am 2. Februar 2005 unterbreitete der Bundesrat dem Parlament deshalb eine Zusatzbotschaft ${ }^{6}$, um die Entsendung von zwei Helikoptern und einem Lufttransportteam von sieben Personen ab Mitte Mai $2005 \mathrm{zu}$ ermöglichen. Das Parlament nahm den Entwurf des zusätzlichen Bundesbeschlusses im März 2005 an.

Die beiden schweizerischen Helikopter (einer für den Einsatz, der andere als Reserve) sind seit Anfang Juni 2005 in Banja Luka stationiert. Für die Schweiz handelt es sich dabei um den vierten Auslandseinsatz mit Militärhelikoptern, aber erst den zweiten Friedenseinsatz nach der Mission für die KFOR im Kosovo $2002^{7}$.

\subsubsection{Friedenskonsolidierungskommission der Vereinten Nationen}

Anlässlich des Weltgipfels der Vereinten Nationen im September 2005 in New York verpflichteten sich die Staaten, vor Jahresende eine Kommission für Frie-

6 Bundesrat, Zusatzbotschaft zum Bundesbeschluss über den Friedensförderungseinsatz von Schweizer Armeeangehörigen in der multinationalen European Union Force (EUFOR) in Bosnien-Herzegowina, vom 2. Februar 2005 (BB1 2005 1603).

7 Die beiden anderen Einsätze verfolgten humanitäre Ziele: Operation ALBA in Albanien (1999) und die Operation in Sumatra nach dem Tsunami (2005).

8 Die Schaffung einer solchen Kommission war ursprünglich im Bericht der Hochrangigen Gruppe über Bedrohungen, Herausforderungen und Wandel (A More Secure World: Our Shared Responsibility, doc. A/59/565) empfohlen worden, der dem Generalsekretär der Vereinten Nationen am 
denskonsolidierung als zwischenstaatliches Beratungsorgan einzusetzen ${ }^{8}$. Das Kernmandat der künftigen Kommission besteht darin, alle interessierten Parteien zur Mobilisierung von Ressourcen, zur Beratung und zur Ausarbeitung von Vorschlägen über integrierte Strategien der Friedenskonsolidierung und der Rehabilitation nach Konflikten zusammenzuführen ${ }^{9}$.

In den Verhandlungen während der 60. Tagung der UN-Generalversammlung bereiteten die Zusammensetzung der Kommission und ihre Unterstellung unter eine übergeordnete Instanz allerdings Schwierigkeiten. Im Februar 2005 hatte die Schweiz im Rahmen der 59. Tagung der Generalversammlung dafür plädiert, die Kommission dem Sicherheitsrat und dem Wirtschafts- und Sozialrat (ECOSOC) der UNO gemeinsam zu unterstellen, um deren als unzureichend angesehene Interaktionen zu verstärken.

Am 20. Dezember 2005 beschlossen der Sicherheitsrat und die UN-Generalversammlung in einer gemeinsamen Resolution ${ }^{10}$, die Kommission für Friedenskonsolidierung als ein beiden Instanzen unterstelltes Organ zu schaffen - ein Präzedenzfall in der Struktur der UNO. Die Kommission soll aus 31 Mitgliedern bestehen, die jeweils für eine verlängerbare Mandatsdauer von zwei Jahren gewählt werden, darunter die fünf ständigen Mitglieder des Sicherheitsrates.

Wie viele andere Länder, die schliesslich den Resolutionstext billigten, meldete die Schweiz Vorbehalte bezüglich der als zu zentral beurteilten Rolle des Sicherheitsrates im operationellen Dispositiv der Kommission an, die hauptsächlich die Aufgabe hat, dem Rat auf Verlangen Stellungnahmen abzugeben ${ }^{11}$. Das von der Schweiz verfochtene Modell war nicht sequentiell, sondern ausgeglichen zwischen Sicherheitsrat und ECOSOC, d.h. zwischen den Bereichen Sicherheit und Entwicklung.

Die Resolution sieht ausserdem die Schaffung eines durch freiwillige Beiträge finanzierten, mehrjährigen ständigen Fonds für die Friedenskonsolidierung nach Konflikten vor $^{12}$.

\subsection{Sicherheitspolitik}

\subsubsection{Organisation für Sicherheit und Zusammenarbeit in Europa (OSZE)}

Die Organisation für Sicherheit und Zusammenarbeit in Europa (OSZE) $)^{13}$ ist ein gesamteuropäisches Sicherheitsorgan, das 1975 gegründet wurde. Ende 2005

1. Dezember 2004 unterbreitet wurde. Diese Empfehlung wurde anschliessend vom UN-Generalsekretär in seinem Bericht In Larger Freedom: Towards Development, Freedom and Human Rights for All vom März 2005 übernommen (doc. A/59/2005), der das Arbeitsdokument der Staats- und Regierungschefs am Weltgipfel bildete.

9 Vereinte Nationen, Informationsdokument über die Friedenskonsolidierungskommission (Peacebuilding Commission), für die Parlamentarische Anhörung 2005 in den Vereinten Nationen erarbeitet, 31. Oktober und 1. November 2005, S. 1.

10 Resolution A/RES/60/180 der Generalversammlung der Vereinten Nationen oder S/RES/1645 (2005) des Sicherheitsrates der Vereinten Nationen.

11 Ibid., Absatz 16.

12 Ibid., Absatz 24.

13 Die OSZE hiess von 1975 bis 1994 „Konferenz für Sicherheit und Zusammenarbeit in Europa“ (KSZE). 
gehörten der Organisation 55 Staaten an, von denen jeder das gleiche politische Gewicht bei der Beschlussfassung besitzt. Die OSZE dient als Forum für politische Verhandlungen und Entscheidungsfindung in den Bereichen Frühwarnung, Konfliktverhütung, Krisenmanagement und Rehabilitation nach Konflikten in der Region ${ }^{14}$. Die Region umfasst - zumindest gemäss der operationellen Dimension der OSZE - Südosteuropa, Osteuropa und Zentralasien.

Die OSZE war seit ihrer Gründung, bis zum UNO-Beitritt der Schweiz, das einzige internationale Forum für Sicherheitspolitik, an dem die Schweiz vollberechtigt mitwirken konnte. Von den drei Dimensionen der Sicherheit ${ }^{15}$, die den Tätigkeitsbereich der OSZE traditionell ausmachen, hat sich die Schweiz seit jeher am meisten für die menschliche Sicherheit interessiert. Die Feldmissionen der $\mathrm{OSZE}^{16}$ bieten zudem wichtige Einsatzgelegenheiten für den Schweizerischen Expertenpool für zivile Friedensförderung (siehe oben, Punkt 11.1.1).

Am 5. und 6. Dezember 2005 tagte der 13. Ministerrat der OSZE in Ljubljana (Slowenien). Die schweizerische Delegation wurde vom Staatssekretär Michael Ambühl geleitet. Nach dessen Auffassung muss die OSZE ihre Strukturen und ihre Funktionsweisen reformieren, da sie eine Krise durchmacht. Drei Hauptgründe erklären diese Krise : Zwischen den Mitgliedsstaaten der OSZE zeichnen sich immer häufiger Trennlinien $a b$; die Koordination der Beschlüsse und der Aktivitäten in den von der OSZE festgelegten drei Dimensionen der Sicherheit ist ungenügend; die Palette der Themen und Aufgaben, mit denen sich die OSZE beschäftigt, weitet sich ständig aus.

Der Reformprozess ist derzeit im Gang (der Ministerrat von Ljubljana hat einen Marschplan festgelegt) und gehört zu den Hauptzielen der belgischen OSZEPräsidentschaft für das Jahr 2006.

\subsubsection{Partnerschaft für den Frieden und Euro-Atlantischer Partnerschaftsrat der NATO}

Am 9. März 2005 nahm der Bundesrat den Jahresbericht 2004 über die Teilnahme der Schweiz am Euro-Atlantischen Partnerschaftsrat (EAPC) und an der Partnerschaft für den Frieden (PfP) $a^{17}$. Laut diesem Bericht beliefen sich die Ausgaben des Eidgenössischen Departements für Verteidigung, Bevölkerungsschutz und Sport (VBS) und des Eidgenössischen Departements für auswärtige Angelegenheiten (EDA) im Rahmen des EAPC und der PfP im Jahr 2004 auf knapp 4 Millionen Franken. Davon werden neun Zehntel vom VBS und ein Zehntel vom EDA bestritten.

Am 2. November 2005 wurde das Individuelle Partnerschaftsprogramm zwischen der Schweiz und der NATO für $2006^{18}$ vorgestellt. Dieses Programm formuliert die Erwartungen der Schweiz an ihre Teilnahme an der Partnerschaft für den Frie-

14 OSZE, OSCE - About, S. 1., auf der Website der OSZE einsehbar unter : <www.osce.org>.

15 Politisch-militärische Sicherheit, ökonomisch-ökologische Sicherheit, menschliche Sicherheit.

16 Es gibt 18 solche Missionen und weitere Feldaktivitäten in 16 Ländern.

17 Bundesrat, Jahresbericht 2004 des Bundesrates über die Teilnahme der Schweiz am Euro-Atlantischen Partnerschaftsrat und an der Partnerschaft für den Frieden, Bern, 9. März 2005, 15 S., auf der Website der Bundesverwaltung über die Partnerschaft für den Frieden einsehbar unter: <www.pfp.admin.ch>.

18 Individuelles Partnerschaftsprogramm zwischen der Schweiz und der NATO für 2006, 2. November 2005, einsehbar unter: <www.pfp.admin.ch> 
den und beschreibt die verfügbaren militärischen und zivilen Ressourcen, die Anwendungsbereiche ihres individuellen Partnerschaftsprogramms sowie die wichtigsten von ihr geplanten Tätigkeiten.

2005 wurde erstmals ein Forum des EAPC über die Sicherheit anberaumt, das die Frühjahrstagung der Aussenminister der Mitgliedsstaaten des Rates ersetzte. Mit dieser Neuorganisation sollen die Arbeitsgruppen des EAPC den Vertretern von NGO und Think-Tanks geöffnet werden, die bei den behandelten Themen eine von der NATO anerkannte Rolle spielen. Das Forum fand am 24. und 25. Mai 2005 in Åre in Schweden statt. Die schweizerische Delegation wurde vom Botschafter Thomas Feller, Chef des Zentrums für Internationale Sicherheitspolitik im EDA, geleitet. Die Diskussionen drehten sich vor allem um die Art der Integration des Balkans in Europa, den Status des Kosovo sowie um den Anschluss von Serbien-Montenegro und Bosnien-Herzegowina an die Partnerschaft für den Frieden. Die Schweiz will den Beitritt der beiden Länder zur PfP unterstützen, sobald sie die gestellten Anforderungen erfüllen, und hat im Übrigen in Åre die Fortsetzung des schweizerischen Engagements auf dem Balkan bekräftigt.

Im Jahr 2005 fanden zwei weitere Tagungen der Mitglieder des EAPC in Brüssel statt, nämlich das Treffen der Verteidigungsminister am 10. Juni und dasjenige der Aussenminister am 8. Dezember. Die Verteidigungsminister befassten sich mit den Folgen der laufenden Reform der NATO, die sich an die aktuellen und künftigen sicherheitspolitischen Herausforderungen anpassen muss, auf die Politiken und Programme des EAPC und der PfP. Die Aussenminister widmeten sich ihrerseits den Friedenssicherungsoperationen der NATO (Afghanistan und Kosovo) und der Frage der Werte innerhalb der PfP.

Daneben organisierte die Schweiz 2005 verschiedene Kurse und Workshops in den traditionellen Bereichen ihres Engagements im Rahmen der Partnerschaft für den Frieden: humanitäres Völkerrecht, Grenzsicherheit, Reform des Sicherheitssektors, Schutz kritischer Infrastruktureinrichtungen und Terrorismusfinanzierung.

\subsection{Kampf gegen Klein- und Leichtwaffen}

\subsubsection{Aktionsprogramm über den illegalen Handel mit Klein- und Leichtwaffen; neues Identifizierungs- und Markierungsinstrument}

Der Kampf gegen Klein- und Leichtwaffen stellt eine komplexe Aufgabe dar, da sich die meisten Staaten auf solche Waffen verlassen, um ihre Sicherheit zu gewährleisten, und ihnen von der UN-Charta (Artikel 51) das Recht auf individuelle und kollektive Selbstverteidigung zuerkannt wird. Erschwerend kommt noch hinzu, dass der Export solcher Waffen mit kommerziellen Interessen verbunden ist. Angesichts der Bilanz von jährlich über einer halben Million Todesfällen, die durch diese Waffen verursacht werden, drei Fünftel davon in den Entwicklungsländern, hatte die Generalversammlung der Vereinten Nationen dennoch im Dezember 1999 beschlossen, eine Konferenz über den illegalen Handel mit Klein- und Leichtwaffen zu organisieren. Diese Konferenz fand im Juli 2001 statt und mündete in ein nicht zwingendes Aktionsprogramm auf

19 United Nations General Assembly, Report of the United Nations Conference on the Illicit Trade in Small Arms and Light Weapons in All Its Aspects, doc. A/CONF.192/15, 20. Juli 2001. 
nationaler, regionaler und globaler Ebene ${ }^{19}$. Das Programm umfasst die kurz zuvor dem Vorbereitungsausschuss der Konferenz unterbreitete schweizerischfranzösische Initiative ${ }^{20}$, welche die Entwicklung eines Instruments zum Ziel hat, das die Rückverfolgbarkeit der Herkunft von Klein- und Leichtwaffen und der Handelsströme gewährleisten soll.

Diese Initiative wurde anlässlich der ersten Zweijahrestagung der Staaten zur Prüfung der Umsetzung des Aktionsprogramms über sämtliche Aspekte der Verhütung, Bekämpfung und Beseitigung des illegalen Klein- und Leichtwaffenhandels, welche im Juli 2003 tagte, als relevant anerkannt. Zudem wurde im Dezember 2003 eine Arbeitsgruppe mit offener Zusammensetzung geschaffen, die beauftragt wurde, einen Entwurf für ein völkerrechtliches Instrument auszuhandeln, das es den Staaten erlauben soll, Leicht- und Kleinwaffen schnell und verlässlich zu identifizieren und zu markieren ${ }^{21}$. Diese vom schweizerischen Botschafter Anton Thalmann geleitete Arbeitsgruppe legte der Generalversammlung der Vereinten Nationen ihren Bericht am 27. Juni 2005 vor $^{22}$.

Vom 11. bis 15. Juli 2005 fand in New York die zweite Zweijahrestagung zur Prüfung des Aktionsprogramms statt. Auf dieser Tagung wurde der Mangel an finanziellen und technischen Ressourcen für die Umsetzung des Aktionsprogramms besonders von den afrikanischen Delegationen bedauert. Der Entwurf für das internationale Instrument zur Identifizierung und Markierung illegaler Klein- und Leichtwaffen im Bericht der Arbeitsgruppe wurde von den Vertretern der Teilnehmerstaaten an der Zweijahrestagung deutlich begrüsst. Zahlreiche Delegationen bedauerten indes, dass dieses Instrument nicht rechtlich bindend sei.

Der stellvertretende Chef der Politischen Abteilung IV des EDA, Stephan Husy, betonte anlässlich der Tagung, dass sich die Erfahrungen der Arbeitsgruppe bei ihren Bemühungen um den Entwurf eines solchen Instruments bei künftigen Initiativen, die das Aktionsprogramm der Vereinten Nationen in bestimmten Bereichen schlagkräftiger gestalten sollen, als wertvoll erweisen werden ${ }^{23}$. Ferner verwies er auf die zentrale Bedeutung der Problematik der Verwendung von Klein- und Leichtwaffen durch nichtstaatliche Akteure, wenngleich diese Frage von der Konferenz im Jahr 2001 wegen ihrer grossen Komplexität nicht offen behandelt worden war. Allerdings ist es gemäss Stephan Husy durchaus möglich, aus dem erfolgreichen Dialog mit diesen Akteuren im Bereich der Personenminen (siehe unten, Punkt 11.4.) auch im Bereich der Klein- und Leichtwaffen Kapital zu schlagen.

Der Entwurf des Instruments wurde von der Generalversammlung der Vereinten Nationen auf ihrer 60. Tagung am 20. Oktober 2005 verabschiedet $^{24}$. Die Reso-

20 United Nations General Assembly, Verbal Note dated $21^{\text {st }}$ March 2001, doc. A/CONF.192/PC/38.

21 United Nations General Assembly, Illicit Trade in Small Arms and Light Weapons in All Its Aspects, doc. A/RES/58/241, 23. Dezember 2003.

22 United Nations General Assembly, Report of the Open-ended Working Group to Negotiate an International Instrument to Enable States to Identify and Trace, in a Timely and Reliable Manner, Illicit Small Arms and Light Weapons, doc. A/60/88, 27. Juni 2005.

23 Erklärung von Stephan Husy in der allgemeinen Aussprache der 2. Zweijahrestagung der Staaten zur Prüfung der Umsetzung des Aktionsprogramms über sämtliche Aspekte der Bekämpfung und Beseitigung des illegalen Klein- und Leichtwaffenhandels, New York, 11. Juli 2005.

24 Generalversammlung der Vereinten Nationen, Dokument des Ersten Ausschusses für Abrüstungs- und internationale Sicherheitsfragen, 20. Oktober 2005, doc. A/C.1/60/L.57. 
lution der Generalversammlung appelliert an alle Staaten, das internationale Instrument umzusetzen, damit die Identifizierung und die Markierung von Klein- und Leichtwaffen Wirklichkeit werden können.

\subsubsection{Small Arms Survey}

Die Schweiz hat das Forschungsprojekt Small Arms Survey, das seit 1999 beim Institut universitaire de hautes études internationales (IUHEI) angesiedelt ist, aus der Taufe gehoben und gehört zu den wichtigsten Geldgebern. Das Hauptziel des Projekts besteht darin, eine neutrale und öffentliche Informationsquelle zu allen Aspekten der Klein- und Leichtwaffen zu bilden. Dieses Projekt beherbergt und verwaltet die Datenbank ${ }^{25}$ der Konferenz der Vereinten Nationen über Klein- und Leichtwaffen (Regierungsdokumente, Abstimmungsergebnisse, Fortschrittsstand bei der Umsetzung des Länderaktionsprogramms, usw.).

Am 11. Juli 2005 - wenige Stunden vor der Eröffnung der zweiten Zweijahrestagung zur Prüfung des Aktionsprogramms über den illegalen Klein- und Leichtwaffenhandel - stellte das Projekt Small Arms Survey in New York sein Jahrbuch 2005 vor $^{26}$, das der unterschätzten Bedeutung der Klein- und Leichtwaffen in bewaffneten Konflikten gewidmet ist. Gemäss dieser Publikation, die ihrerseits als ein Werkzeug im Kampf gegen die unkontrollierte Verbreitung und den Missbrauch von Klein- und Leichtwaffen angesehen werden kann, kommen 60 bis 90 Prozent der Todesopfer in bewaffneten Auseinandersetzungen durch solche Waffen um. Das erste Jahrbuch des Small Arms Survey war vor der UN-Konferenz über sämtliche Aspekte des illegalen Handels mit Klein- und Leichtwaffen im Jahr 2001 vorgestellt worden.

\subsubsection{In-Kraft-Treten des Protokolls gegen die illegale Herstellung von und den illegalen Handel mit Schusswaffen}

Das Protokoll gegen die unerlaubte Herstellung von Schusswaffen, dazugehörigen Teilen und Komponenten und Munition und gegen den unerlaubten Handel damit - eine Ergänzung des Übereinkommens der Vereinten Nationen gegen die grenzüberschreitende organisierte Kriminalität - trat am 3. Juli 2005 nach der 40. Ratifizierung in Kraft. Das Übereinkommen war von der Generalversammlung der Vereinten Nationen am 31. Mai 2001 angenommen worden.

Während der gesamten Verhandlungen über das internationale Instrument zur Identifizierung und Markierung von illegalen Kleinwaffen (militärischen Typs siehe oben, Punkt 11.3.1) wurde gewährleistet, dass dieses Instrument und das Protokoll gegen die illegale Herstellung von und den illegalen Handel mit Schusswaffen sich gegenseitig ergänzen. Anders als das erste Instrument ist das Protokoll für die Parteien rechtsverbindlich.

Die seit Januar 1999 zu dieser Schusswaffenart laufenden Verhandlungen sind als „Prozess von Wien“ bekannt, weil alle Tagungen des Ad-hoc-Aus-

25 Es handelt sich um eine öffentliche, für alle interessierten Personen zugängliche Datenbank: $<$ www.smallarmssurvey.org/databases.htm >.

26 Small Arms Survey, Small Arms Survey 2005, Oxford, Oxford University Press, 2005, 352 S. 
schusses, der mit der Ausarbeitung des Übereinkommens und der drei Protokolle $^{27}$ beauftragt ist, in Wien stattfanden. Seit dem In-Kraft-Treten des Übereinkommens wurden die Parteien zu zwei weiteren Konferenzen in Wien eingeladen, wobei die erste vom 28. Juni bis 9. Juli 2004, die zweite vom 10. bis 21. Oktober 2005 tagte.

Die Schweiz hat das Übereinkommen am 12. Dezember 2000 unterzeichnet, es aber noch nicht ratifiziert. Der Bundesrat hat den Eidgenössischen Räten am 26. Oktober 2005 eine Botschaft über die Genehmigung dieses Übereinkommens und der beiden zusätzlichen Protokolle unterbreitet ${ }^{28}$; dasjenige über die Schusswaffen ist nicht Gegenstand der Botschaft. Das Übereinkommen ist am 29. September 2003 in Kraft getreten und zählte im Jahr 2005110 Parteien. Die Schweiz hat das Protokoll, dem 200544 Mitglieder angehörten, noch nicht ratifiziert. Anlässlich der ersten Parteienkonferenz im Jahr 2004 zählte das Übereinkommen erst 79 und das Protokoll 21 Parteien: Offensichtlich messen die Länder diesen Instrumenten eine wachsende Bedeutung bei. Die Schweiz ist noch nicht Partei dieser Instrumente, da auf nationaler Ebene die erforderlichen Gesetzesgrundlagen für die Ratifizierung des Übereinkommens und des Zusatzprotokolls bislang fehlen.

\subsection{Personenminen, sonstige Landminen und explosive Kriegsmunitionsrückstände}

\subsubsection{Tagung der Vertragsparteien der Ottawa-Konvention über das Verbot von Personenminen (Zagreb, 28. November - 2. Dezember 2005)}

Die Konvention von Ottawa über das Verbot des Einsatzes, der Lagerung, der Herstellung und der Weitergabe von Anti-Personenminen und über deren Vernichtung ist im März 1999 in Kraft getreten. Das Übereinkommen soll den durch Personenminen verursachen Leiden ein Ende setzen. Dazu verfolgt es vier Hauptziele: Beitritt aller Staaten zur Konvention, Vernichtung der Personenminenbestände, Räumung verminter Bereiche und Hilfeleistung für die Opfer. Um diese Ziele zu erreichen, verabschiedeten die Konventionsparteien anlässlich der ersten Konferenz Ende 2004 in Nairobi einen Aktionsplan mit 70 Punkten.

[D] Jahrbuch 2005, Nr. 1, Kapitel 11, 11.3.2. Konferenz zur Überprüfung der Ottawa-Konvention, S. 187-188.

Die sechste Jahrestagung der Vertragsparteien der Ottawa-Konvention fand vom 28. November bis zum 2. Dezember 2005 in Zagreb statt. Die Vertragsstaaten, darunter die Schweiz, erhielten erstmals formell Gelegenheit, die bei der Umsetzung des bis 2009 laufenden Aktionsplans von Nairobi geleisteten Fortschritte zu

27 Protokoll zur Verhinderung und Bestrafung des Menschenhandels, insbesondere des Frauen- und Kinderhandels; Protokoll gegen die Schlepperei auf dem Land-, See- und Luftweg; Protokoll gegen die unerlaubte Herstellung von Schusswaffen, dazugehörigen Teilen und Komponenten und Munition und gegen den unerlaubten Handel damit.

28 Bundesrat, Botschaft über die Genehmigung des UNO-Übereinkommens gegen die grenzüberschreitende organisierte Kriminalität, des Zusatzprotokolls zur Verhinderung und Bestrafung des Menschenhandels, insbesondere des Frauen- und Kinderhandels, und des Zusatzprotokolls gegen die Schlepperei auf dem Land-, See- und Luftweg vom 26. Oktober 2005 (BB1 2005 6693). 
messen. Diese Fortschritte sind Teil des Berichts, den die Vertragsparteien anlässlich ihrer Tagung angenommen haben ${ }^{29}$. Der Bericht zieht die Bilanz der bisherigen Leistungen und definiert die Prioritäten für die Umsetzung des Aktionsplans bis zur siebten Parteienkonferenz im Jahr 2006. Es wurde beschlossen, dass jede Tagung bis zur zweiten Konferenz zur Überprüfung der Konvention, die für 2009 geplant ist, einen entsprechenden Bericht ausarbeiten soll. Auf politischer Ebene haben die Vertragsparteien mit der Annahme der Erklärung von Zagreb ihren Willen bekräftigt, die Ziele des Übereinkommens durch die Umsetzung des Aktionsplans zu erreichen.

Der Leiter der Schweizer Delegation an der Tagung in Zagreb, Botschafter Jürg Streuli, verwies nachdrücklich auf die von der Schweiz seit drei Jahren geleistete Reflexionsarbeit über die Möglichkeiten der Staaten, bewaffnete nichtstaatliche Akteure zum Verzicht auf die Verwendung von Personenminen zu bewegen. Diese spezifische Frage wird in einem der 70 Punkte des Aktionsplans von Nairobi behandelt ${ }^{30}$. Die ersten Antwortansätze, ausgehend von den Überlegungen der Schweiz, empfehlen, dass die in der Anti-Minen-Bewegung aktiven Regierungs- und Nichtregierungsorganisationen eine Zugangsbewilligung zu verminten Zonen, die von nichtstaatlichen Akteuren kontrolliert werden, erhalten sollen, und fordern alle beteiligten Akteure auf, die zur Förderung der Umsetzung dieser Massnahme des Nairobi-Plans erforderlichen Kontakte zu ermöglichen.

Zur Vorbereitung auf die Konferenz fand im Oktober 2004 ein Seminar in Montreux statt ${ }^{31}$. An den informellen Diskussionen des Expertenseminars auf Einladung der Politischen Abteilung IV des EDA beteiligten sich eine Gruppe interessierter Staaten, eine begrenzte Zahl geladener Experten sowie ehemalige Mitglieder von nichtstaatlichen bewaffneten Gruppen, die ein Schlaglicht aus der Praxis auf die Lektionen der Vergangenheit und die Zukunftsperspektiven warfen.

\subsubsection{Erste Tagung der Unterzeichner der Verpflichtungsurkunde des Appells von Genf, ,Deed of Commitment" (Genf, 31. Oktober - 2. November 2004)}

Beim im März 2000 gegründeten Appell von Genf (Geneva Call) handelt es sich um eine internationale humanitäre Organisation, die das Ziel verfolgt, nichtstaatliche bewaffnete Akteure in das Personenminenverbot einzubeziehen. Der Appell von Genf ist ein strategischer Partner der Politischen Abteilung IV des EDA und wird von dieser finanziell unterstützt ${ }^{32}$.

Wie die Vertragsparteien der Ottawa-Konvention (siehe oben) verpflichten sich die nichtstaatlichen Akteure mit der Unterzeichnung der Urkunde des Appells

29 Zagreb Progress Report, einsehbar auf der Website des Internationalen Zentrums für humanitäre Minenräumung in Genf, <www.gichd.ch>.

30 Aktion 46: „Continue to support, as appropriate, mine action to assist affected populations in areas under the control of armed non-state actors, particularly in areas under the control of actors which have agreed to abide by the Convention's norms".

31 The role of states in the universal ban of anti-personnel mines in internal armed conflicts - Expert Workshop on bringing armed groups into a process to ban anti-personnel mines.

32 Die Europäische Kommission leistet ebenfalls einen wesentlichen Beitrag an den Appell von Genf. 
von Genf, „Deed of Commitment“, auf die Verwendung, Herstellung, Lagerung und Weitergabe von Personenminen zu verzichten. Der Appell von Genf berät diese Akteure anschliessend bei ihrem Minenräumungsprogramm und fordert von ihnen eine regelmässige Berichterstattung über die ergriffenen Massnahmen.

Im November 2005 hatten 28 nichtstaatliche Bewegungen die Urkunde „Deed of Commitment" unterzeichnet, zuletzt der Front Polisario in der WestsaharaRegion (3. November 2005). Die Präsidentin des Appells von Genf, Elisabeth Reusse-Decrey, äusserte die Hoffnung, dass dieses Engagement des Front Polisario Marokko zum Beitritt zur Ottawa-Konvention bewegen möge.

Vom 31. Oktober bis 2. November - zeitgleich mit dem Gipfel von Nairobi für eine Welt ohne Minen ${ }^{33}$ - fand in Genf die erste Tagung der Unterzeichner der Verpflichtungsurkunde „Deed of Commitment“ statt, die vom Appell von Genf ausgerichtet wurde. Der Bericht der Tagung erschien im September $2005^{34}$. Der Appell von Genf erhoffte sich von der Tagung Antworten auf mehrere Fragen, besonders die Identifizierung der Herausforderungen, mit denen die Unterzeichner bei der Erfüllung ihrer Verpflichtungen konfrontiert sind, und die Gegenüberstellung der Argumente für bzw. wider eine Erweiterung des Mandats des Appells von Genf auf andere humanitäre Standards.

Hinsichtlich der Herausforderungen zeigte die Tagung, dass die finanzielle und technische Unterstützung für die Unterzeichnerbewegungen eine Schlüsselrolle spielt, damit sie ihre Minenräumungsprogramme erfolgreich durchführen können. Ausserdem wurde deutlich, dass der Appell von Genf den Schwerpunkt seines Diskurses darauf legen muss, dass die Unterzeichnung der Verpflichtungsurkunde (,Deed of Commitment“) auf rein humanitären Erwägungen zu beruhen hat - unabhängig von Argumenten der Gegenseitigkeit zwischen den Konfliktparteien bei der Verwendung von Personenminen: Solche Argumente werden von den Bewegungen häufig angeführt, um Misserfolge bei der Umsetzung ihrer Verpflichtungen aus der Unterzeichnung des „Deed of Commitment“ zu rechtfertigen, oder auch von anderen Bewegungen, um ihre Ablehnung der Unterzeichnung dieser Urkunde zu begründen.

In der Frage der Erweiterung des Mandats des Appells von Genf auf andere humanitäre Normen (z.B. Kindersoldaten, Folter oder sonstige laut humanitärem Völkerrecht verbotene Handlungen) gingen die Auffassungen der Tagungsteilnehmer auseinander. Eine etwaige Ausdehnung des Mandats des Appell von Genf darf nicht auf Kosten des aktuellen Mandats im Bereich der Minen erfolgen; zudem sollten diejenigen Organisationen, die sich bereits mit den von einer Erweiterung berührten Bereichen befassen, unbedingt konsultiert werden.

\subsubsection{Botschaft betreffend das Protokoll über explosive Kriegsmunitionsrückstände}

Der Bundesrat unterbreitete dem Parlament am 17. August 2005 die Botschaft zum Entwurf des Bundesbeschlusses betreffend das Protokoll über explosive

33 Daran beteiligten sich die Vertragsparteien der Ottawa-Konvention zur ersten Überprüfungskonferenz (siehe Schweizerisches Jahrbuch für Entwicklungspolitik 2005, Nr. 1, S. 187).

34 Geneva Call, An Inclusive Approach to Armed Non-State Actors and International Humanitarian Norms: Report of the First Meeting of Signatories to Geneva Call's Deed of Commitment, September 2005, 62 S., einsehbar auf der Website des Appells von Genf unter: <www.genevacall.org > > resources. 
Kriegsmunitionsrückstände als Anhang der Konvention von 1980 über bestimmte konventionelle Waffen (Protokoll V) ${ }^{35}$. Die Schweiz hat die Rahmenkonvention (einschliesslich der Änderung von Artikel 1) sowie die vier Protokolle bereits ratifiziert.

Das neue Protokoll soll mit der Verpflichtung der Staaten, postkonfliktuelle Abhilfemassnahmen zu ergreifen, die Gefahren und die negativen Auswirkungen explosiver Kriegsmunitionsrückstände für die Zivilbevölkerung auf ein Mindestmass beschränken.

Gemäss der Botschaft des Bundesrates hat die Schweiz sich während der Verhandlungen zum Zusatzprotokoll über die explosiven Kriegsmunitionsrückstände (Explosive Remnants of War - ERW) ,für ein substanzielles, effizientes und rechtsverbindliches Protokoll eingesetzt, das die negativen Auswirkungen explosiver Kriegsmunitionsrückstände, einschliesslich Streumunition, auf die Zivilbevölkerung und den Wiederaufbau des Landes vermindert"36. Die Verhandlungen mündeten in Massnahmen, die sich aus der Berücksichtigung humanitärer wie auch militärischer Bedürfnisse ergeben. Das Protokoll V löst zwar nicht alle Probleme im Zusammenhang mit explosiven Kriegsmunitionsrückständen, stellt aber dennoch eine wichtige Anerkennung der Verantwortung der Staaten gegenüber der Zivilbevölkerung für die verheerenden Auswirkungen bestimmter Munitionsarten dar. Bisher sah das Völkerrecht keine spezifischen Regeln für explosive Kriegsmunitionsrückstände vor. Das In-Kraft-Treten des Protokolls, das elf rechtlich verbindliche Artikel und einen fakultativen technischen Anhang umfasst, wird somit, in seiner aktuellen Form, eine Stärkung des humanitären Völkerrechts bilden. Die Diskussionen zwischen den Vertragsparteien des Übereinkommens über konventionelle Waffen zum Thema Munition, einschliesslich Submunition, die zu explosiven Kriegsmunitionsrückständen werden kann, sind jedoch mit der Annahme des Protokolls V noch nicht abgeschlossen. Die Schweiz engagiert sich weiterhin tatkräftig für einen besseren Schutz der Zivilbevölkerung gegen bestimmte Munitionsarten.

Das Protokoll V entspricht den Zielen der schweizerischen Minenräumungspolitik, die heute bereits die Entfernung der explosiven Kriegsmunitionsrückstände umfasst.

Am 24. Oktober 2005 beantragte die Sicherheitspolitische Kommission des Ständerates einstimmig die Annahme des Entwurfs des Bundesrates. Der Ständerat schloss sich in der Wintersession am 15. Dezember 2005 dem Antrag seiner Kommission an. Der Nationalrat wird sich 2006 damit befassen.

Die Annahme des Entwurfs des Bundesbeschlusses durch das Parlament wird es der Schweiz erlauben, die Ratifikationsurkunden des Protokolls, das bislang noch nicht in Kraft getreten ist ${ }^{37}$, beim Generalsekretariat der Vereinten Nationen zu hinterlegen.

35 Bundesrat, Botschaft betreffend das Protokoll über explosive Kriegsmunitionsrückstände vom 28. November 2003 des Übereinkommens vom 10. Oktober 1980 über das Verbot oder die Beschränkung des Einsatzes bestimmter konventioneller Waffen, die übermässige Leiden verursachen oder unterschiedslos wirken können (Protokoll V), vom 17. August 2005 (BB1 2005 5579).

36 Ibid., S. 5586.

37 Im November 2005 hatten 13 Staaten zugesagt, sich durch das Protokoll V zu binden, und es braucht 20 Staaten, damit es in Kraft tritt (Quelle: <http://disarmament.un.org/TreatyStatus.nsf> >CCWC $>$ Protocol V). 
Am 7. Dezember 2005 wurde eine parlamentarische Initiative über eine spezifische Regelung der Submunition in der Schweiz eingereicht ${ }^{38}$. Die Initiative verlangt eine Änderung des Bundesgesetzes über das Kriegsmaterial, genauer das Verbot, Streumunitionen herzustellen oder zu erwerben, ausser zur Entwicklung von Such-, Räumungs- und Vernichtungsverfahren für solche Waffen.

\subsection{Massenvernichtungswaffen}

\subsubsection{Internationales Übereinkommen zur Bekämpfung des Nuklearterrorismus}

Das internationale Übereinkommen zur Bekämpfung des Nuklearterrorismus ${ }^{39}$ wurde von der Generalversammlung der Vereinten Nationen am 13. April 2005 nach sieben Jahren Verhandlungen, an denen die Schweiz aktiv mitgewirkt hatte, verabschiedet. Der UN-Generalsekretär Kofi Annan hatte in seinem Bericht vom 21. März $2005^{40}$ an die Staaten appelliert, diese Konvention - die erste seit den Anschlägen vom 11. September 2001 - abzuschliessen.

Das Übereinkommen wurde vom Bundesrat am 22. Juni 2005 genehmigt und vom Bundespräsidenten Samuel Schmid am 14. September 2005 während des UNO-Weltgipfels in New York unterzeichnet. „Mit der Unterzeichnung des Übereinkommens setzt die Schweiz ein klares Zeichen für die Bekämpfung eines der abscheulichsten Verbrechen überhaupt."41

Im November 2005 hatten 92 Staaten das Übereinkommen unterzeichnet, aber noch kein Staat hatte es ratifiziert. Damit das Übereinkommen in Kraft tritt, muss es von 22 Staaten ratifiziert werden. Diese verpflichten sich damit zur Bestrafung des Besitzes oder der Verwendung von Nuklearmaterial mit dem Ziel, Menschen zu verletzen oder zu töten, Sachschäden anzurichten oder die Umwelt zu beeinträchtigen.

\subsubsection{Konferenz zur Überprüfung des Atomwaffensperrvertrags (New York, 2.-27. Mai 2005)}

Der Atomwaffensperrvertrag (Non-Proliferation Treaty), der seit dem 5. März 1970 in Kraft ist, beruht auf dem Verzicht der atomwaffenlosen Vertragsstaaten auf solche Waffen (Non-Proliferation), und im Gegenzug auf der nuklearen Abrüstung der Staaten mit Atomwaffen. Der Vertrag wird alle fünf Jahre von den Staaten, die ihn ratifiziert haben ${ }^{42}$, anlässlich der Überprüfungskonferenzen untersucht. Dabei stellen die Vertragsparteien sicher, dass sie die zur Verwirkli-

38 „05.452. Parlamentarische Initiative. Revision des Bundesgesetzes über das Kriegsmaterial“, eingereicht von John Dupraz beim Nationalrat am 7. Dezember 2005, <www.parlament.ch>.

39 Generalversammlung der Vereinten Nationen, Resolution A/RES/59/290, 13. April 2005.

40 United Nations, In Larger Freedom: Towards Development, Security and Human Rights vor All, Bericht des UN-Generalsekretärs, doc. A/59/2005, 21. März 2005.

41 EDA, „Bundesrat genehmigt Übereinkommen zur Bekämpfung des Nuklearterrorismus“, Pressemitteilung, 22. Juni 2005.

42 Es handelt sich um 189 Staaten (November 2005). Die Schweiz hat den Vertrag am 27. September 1969 unterzeichnet und am 9. März 1977 ratifiziert (Quelle: <http://disarmament.un.org/TreatyStatus.nsf > > NPT). 
chung der Vertragsziele erforderlichen Massnahmen ergreifen, und erlassen Empfehlungen, um sich auf diesem Weg zu engagieren. Ein Vorschlag Kanadas, über den zurzeit noch verhandelt wird und dem sich die Schweiz anschliesst, sieht vor, jährliche Konferenzen abzuhalten, um bei Vertragsverletzungen rascher reagieren zu können.

Die siebte Überprüfungskonferenz des Atomwaffensperrvertrags tagte vom 2. bis 27. Mai 2005 in New York in Krisenstimmung - wegen des Rücktritts Nordkoreas vom Vertrag im Jahr 2003, wegen der Ungewissheit über den wirklichen Charakter des iranischen Atomprogramms (trotz der seit 2003 von der Internationalen Atomenergieagentur (IAEA) durchgeführten Ermittlungen), und wegen der Aufdeckung eines Kerntechnologie-Schwarzmarkts im Jahr 2004.

In der allgemeinen Aussprache der Konferenz, vor der Arbeit der Ausschüsse, äusserte der ständige Vertreter der Schweiz bei den Vereinten Nationen, Peter Maurer, Besorgnis bezüglich dieser Themen, beklagte aber auch, dass bestimmte Kernwaffen besitzende Staaten den Aufbau ihres Arsenals mit weiteren Krediten vorantrieben, und bedauerte, dass das Ziel der Universalität des Atomwaffensperrvertrags noch nicht errreicht sei, da Indien, Pakistan und Israel dem Vertrag immer noch nicht beigetreten seien. Dagegen bestätigte er, dass der Atomwaffensperrvertrag für die Schweiz das einzige globale, umfassende und rechtsverbindliche Instrument zur Förderung der atomaren Nichtverbreitung und Abrüstung bleibe ${ }^{43}$ und dass es wesentlich sei, die Errungenschaften der vorherigen Überprüfungskonferenzen zu bewahren ${ }^{44}$.

Anlässlich der Überprüfungskonferenz von 1995 wurden Grundsätze und Ziele für die atomare Nichtverbreitung und Abrüstung angenommen, welche die Vertragsstaaten mit Atomwaffen verpflichten, ihre Abrüstung zu verwirklichen. Die darauffolgende Überprüfungskonferenz vom Jahr 2000 verfolgte diesen Weg weiter und erarbeitete ein Dokument mit 13 praktischen Etappen für die Umsetzung der atomaren Abrüstung. Die Konferenz von 2005 führte nicht zur einer Abschlusserklärung - aus den oben erwähnten Gründen, aber auch wegen der Haltung der Vereinigten Staaten, die anstatt abzurüsten, neue strategische Kernwaffen entwickeln ${ }^{45}$, und die im Jahr 2003 die „Proliferation Security Initiative“ (PSI) ${ }^{46}$ lancierten, deren Ziele sich mit denjenigen des Atomwaffensperrvertrags über die Nichtweiterverbreitung decken.

43 Ständige Vertretung der Schweiz bei den Vereinten Nationen, Erklärung von Botschafter Peter Maurer, ständiger Vertreter der Schweiz bei den Vereinten Nationen in New York, anlässlich der Überprüfungskonferenz 2005 der Vertragsparteien des Atomwaffensperrvertrags in New York, 3. Mai 2005, S. 1.

44 Ibid., S. 3.

45 „Donald Rumsfeld et le Pentagone n’ont pas renoncé au ,,brise-bunker“, Le Temps, 3. Mai 2005.

46 Website der Initiative : <www.proliferationsecurity.info>. 


\section{QUELLEN}

Bundesrat, Jahresbericht 2004 des Bundesrates über die Teilnahme der Schweiz am Euro-Atlantischen Partnerschaftsrat und an der Partnerschaft für den Frieden, Bern, 9. März 2005, 15 S.

Erklärung von Botschafter Peter Maurer, ständiger Vertreter der Schweiz bei den Vereinten Nationen, anlässlich der 59. Tagung der Generalversammlung, Punkte 45 und 55 der Tagesordnung, 21. April 2005.

Eidgenössisches Departement für auswärtige Angelegenheiten (EDA), Bericht 2004 über Massnahmen zur zivilen Konfliktbearbeitung und Menschenrechtsförderung, Bern, 18. Mai 2005, 21 S.

Neue Zürcher Zeitung, „Höchste Zeit für einen Atomkonsens“, 4.-5. Juni 2005.

\section{INTERNET-ADRESSEN}

Appell von Genf (Geneva Call) : <www.genevacall.org $>$.

Genfer Zentrum für Sicherheitspolitik (GCSP) : <www.gcsp.ch>.

Internationales Zentrum für humanitäre Minenräumung, Genf (GICHD) : <www.gichd.ch>.

Genfer Zentrum für die demokratische Kontrolle der Streitkräfte (DCAF) : <www.dcaf.ch $>$.

Eidgenössisches Departement für auswärtige Angelegenheiten (EDA) : <www.eda.admin.ch >

Eidgenössisches Departement für Verteidigung, Bevölkerungsschutz und Sport (VBS) : $<$ www.vbs.admin.ch $>$.

Schweizerische Mission bei der NATO (Partnerschaft für den Frieden) : <www.nato.int/pfp/ch> .

Organisation für Sicherheit und Zusammenarbeit in Europa (OSZE) : <www.osce.org>.

Partnerschaft für den Frieden, Website der Bundesverwaltung „Die Schweiz und die Partnerschaft für den Frieden": <www.pfp.admin.ch>.

Small Arms Survey: <www.smallarmssurvey.org $>$.

United Nations Mine Action Service (UNMAS) : <www.mineaction.org >. 